\title{
Study of $D^{*+}$ and search for $D^{* * 0}$ production by neutrinos in BEBC
}

\author{
Big Bubble Chamber Neutrino Collaboration
}

\begin{abstract}
A.E. Asratyan ${ }^{5}$, M. Aderholz ${ }^{6}$, V.V. Ammosov ${ }^{4}$, G.S. Gapienko ${ }^{4}$, V.A. Gapienko ${ }^{4}$, J. Guy ${ }^{7}$, G.T. Jones ${ }^{1}$, V.S. Kaftanov ${ }^{5}$, U.F. Katz ${ }^{6 *}$, J. Kern ${ }^{6}$, V.A. Korotkov ${ }^{4}$, S.P. Krutchinin ${ }^{5}$, M.A. Kubantsev ${ }^{5}$, P. Marage ${ }^{2}$, D.R.O. Morrison ${ }^{3}$, N. Schmitz ${ }^{6}$, W. Venus ${ }^{7}$, W. Wittek ${ }^{6}$, V.G. Zaetz ${ }^{4}$
\end{abstract}

\begin{abstract}
Data from BEBC experiments are combined to provide large statistics for neutrino interactions. Charged $D^{*}$ mesons are produced in $(1.22 \pm 0.25) \%$ of neutrino and $(1.01 \pm 0.31) \%$ of antineutrino charged current interactions. The mean fraction of the hadronic laboratory energy taken by the $D^{*+}$ in these events is $0.59 \pm 0.03 \pm 0.08$. Less than $18 \%$ of all charged $D^{*}$ mesons from (anti)neutrino interactions are found to be daughters of $D^{* * 0}$ (at the $90 \%$ confidence level).
\end{abstract}

[1] University of Birmingham, Birmingham B15 2TT, UK.

[2] Inter-University Institute for High Energies, ULB-VUB, B-1050 Brussels, Belgium.

[3] CERN, CH-1211 Geneva 23, Switzerland.

[4] Institute of High Energy Physics, RU-142284, Protvino, Moscow Region, Russia.

[5] Institute of Theoretical and Experimental Physics, B. Cheremushkinskaya, 25; RU-117259 Moscow, Russia.

[6] Max-Planck-Institut für Physik, 80805 München, Germany.

[7] Rutherford Appleton Laboratory, Chilton, Didcot, OX11 0QX, UK.

* Now at Physikalisches Institut, Universität Bonn, 53115 Bonn, Germany.

To be submitted to Zeitschrift für Physik C 


\section{Introduction.}

This paper studies inclusive production of charmed vector mesons, $D^{*+}(2010)$, in neutrino and antineutrino charged current interactions. The observed $D^{*+}$ mesons are then used to search for production of orbitally excited $c \bar{u}$ states, the $D^{* * 0}$ mesons ${ }^{1}$. The existing neutrino data on $D^{*+}$ are restricted to a few bubble chamber experiments with limited statistics [1]-[3], while the $D^{* * 0}$ has not previously been investigated. However, we observed the production of the strange counterpart of the $D^{* *}$ states, the $D_{s}^{* *}(2536)$ meson, by neutrinos[4]. Strong decays of two distinct $D^{* * 0}$ meson states

$$
\begin{aligned}
D^{* * 0}\left(1^{+}\right) & \rightarrow D^{*+}(2010) \pi^{-} \\
D^{* * 0}\left(2^{+}\right) & \rightarrow D^{*+}(2010) \pi^{-} \\
& \rightarrow D^{+}(1869) \pi^{-}
\end{aligned}
$$

with respective masses near 2420 and $2460 \mathrm{MeV}$ and both widths around $20 \mathrm{MeV}$ have been observed in $\mathrm{e}^{+} \mathrm{e}^{-}$and photoproduction experiments (see [5] and references therein).

This study combines the data of three neutrino experiments using the bubble chamber BEBC filled with hydrogen and with deuterium (the light fills) and with a heavy neon-hydrogen mixture. The three CERN experiments WA21 [6], WA25 [7], and WA59 [8] used a very similar wide-band horn-focussed beam, with mean neutrino- and antineutrino-induced event energies near 53 and $40 \mathrm{GeV}$, respectively. The combined charged current sample of the three experiments is some 52500 neutrino and 42700 antineutrino events with muon momentum over 5 $\mathrm{GeV} / \mathrm{c}$ and neutrino energy over $10 \mathrm{GeV}$, as shown in table 1 .

As the secondary vertices from weak decays of charmed particles are not resolved in the bubble chamber, one has to rely on the combinatorial mass selection of their decay products usually involving strange particles. Note that while the neutral kaons are identified in the bubble chamber by the $K_{s}^{0} \rightarrow \pi^{+} \pi^{-}$decays, the charged kaons are, on the whole, indistinguishable from pions. The kaon hypothesis is tried combinatorially for all charged particles unless identified otherwise.

2. Selection of $D^{0}$ and $D^{*+}$ candidates.

The $D^{*+}(2010)$ decay

$$
D^{*+}(2010) \rightarrow D^{0}(1864) \pi^{+}
$$

with subsequent $D^{0}$ meson weak decays

$$
\begin{aligned}
D^{0} & \rightarrow K_{s}^{0} \pi^{+} \pi^{-} \\
& \rightarrow K^{-} \pi^{+} \\
& \rightarrow K^{-} \pi^{+} \pi^{-} \pi^{+}
\end{aligned}
$$

is selected by triggering on the near-threshold kinematics (or slow pion) of the $D^{*+} \rightarrow D^{0} \pi^{+}$ decay.

If the candidate $D^{0}$ decay products are called $X^{0}$, then the candidate is selected when the measured mass difference

$$
r=m\left(X^{0} \pi^{+}\right)-m\left(X^{0}\right)-m\left(\pi^{+}\right)
$$

is within 2.5 standard deviations of that for $D^{*+} \rightarrow D^{0} \pi^{+}(5.85 \mathrm{MeV})$. Both the experimental error on $r, \sigma_{r}$, and the phase space increase steeply toward higher $r$ values, resulting in a

\footnotetext{
${ }^{1}$ Charge-conjugate states are implied throughout this paper for production by antineutrinos.
} 
Table 1 The numbers of charged current events used after the selections for the muon and neutrino energy.

\begin{tabular}{|c|c|c|c|}
\hline experiment & target & neutrinos & antineutrinos \\
\hline WA21 & hydrogen & 17910 & 12414 \\
WA25 & deuterium & 25171 & 15467 \\
WA59 & neon & 9420 & 14846 \\
\hline
\end{tabular}

high level of combinatorial background from the high- $r$ region. To reduce the latter, for $r$ values in excess of $5.85 \mathrm{MeV}, \sigma_{r}$ is computed after rescaling the slow pion 3-momentum to fit the correct value of the mass difference. (The rescaling effectively fixes the $\pi^{+}$momentum in the $D^{0}$ frame but not its angles there. However, we have verified that the 'rescaled' error on $r$ is virtually insensitive to these angular degrees of freedom. For definiteness, the rescaling procedure used therefore does not change the $\pi^{+}$angle in the $D^{0}$ rest frame.) The error on $r$ is thereby significantly reduced, as is the combinatorial background (by a factor of some 1.4 for the combined event sample). A posteriori, this procedure is justified by non-observation of a significant leakage of the signal into the mass-difference sidebands (see Fig 1e below).

When $X^{0}$ is a $K^{-} \pi^{+}$or $K^{-} \pi^{+} \pi^{-} \pi^{+}$combination, then those combinations with the $K^{-}$ emitted in very forward or backward directions in the $X^{0}$ rest frame with respect to its boost direction from the laboratory system, $|\cos \theta|$ above 0.9 , are dropped. The $D^{0}$ being spinless, the acceptance of this cut is 0.9 . When $X^{0}$ is $K_{s}^{0} \pi^{+} \pi^{-}$, the mass of the $K^{0} \pi^{-}$is required to be within $\pm 60 \mathrm{MeV}$ of the $K^{*-}$ central mass value. When $X^{0}$ is $K^{-} \pi^{+} \pi^{-} \pi^{+}$, the highermass $\pi^{+} \pi^{-}$pair is required to be in the $\rho$ mass region of $0.70-0.85 \mathrm{MeV}$ (corresponding to the width of the $\rho$ resonance). From ref. [9], we estimate that the acceptance of this selection is near 0.47. Even with this tight selection based on the observed resonant structure of the $D^{0} \rightarrow K^{-} \pi^{+} \pi^{-} \pi^{+}$decay, the very high combinatorial background in this channel necessitates a strong cut on the $D^{*}$ fractional hadronic energy (see below).

On the other hand, the precision on $m\left(X^{0}\right)$ for the $K^{-} \pi^{+} \pi^{-} \pi^{+}$channel (about $\pm 19 \mathrm{MeV}$ on average) is better than in the two others (about $\pm 29 \mathrm{MeV}$ ). For the light-fill data only the corresponding precisions are \pm 16 and $\pm 24 \mathrm{MeV}$, respectively. The mean uncertainty on the mass difference $r$ is about $\pm 1.1 \mathrm{MeV}$ for all the data and $\pm 0.8 \mathrm{MeV}$ for the light-fill data only. To improve these mass resolutions, only final state charged particles (or $K_{s}^{0}$ ) with fractional momentum error below 0.1 have been used for the precisions quoted and throughout the analyses below: a small correction for this loss is made when appropriate.

\section{3. $D^{*+}$ production.}

The mass distributions of the selected $X^{0}=K^{-} \pi^{+}$and $K_{s}^{0} \pi^{+} \pi^{-}$combinations tagged by a slow pion (i.e. passing the mass-difference selection with an additional $\pi^{+}$) are plotted in Fig 1. An enhancement is indeed observed near the $D^{0}(1864)$ mass.

The 'detachment' is the mass difference divided by the computed uncertainty on $m\left(X^{0}\right)$, $\left[m\left(X^{0}\right)-m\left(D^{0}\right)\right] / \sigma_{m}$, plotted in Fig 2a which verifies that the width of the peak is compatible with the experimental resolution. No enhancements are observed in the $m\left(X^{0}\right)$ distributions with either a wrong-sign slow pion (Fig 1d), or with a right-sign pion in the mass difference $r$ sidebands between 2.5 and 5 standard deviations (Fig 1e). As expected, the width of the $D^{0}$ peak is reduced as soon as only light-fill data are considered, the shaded entries in the figure.

To gain an insight into the dynamics of $D^{*+}$ production, we then correct the visible 

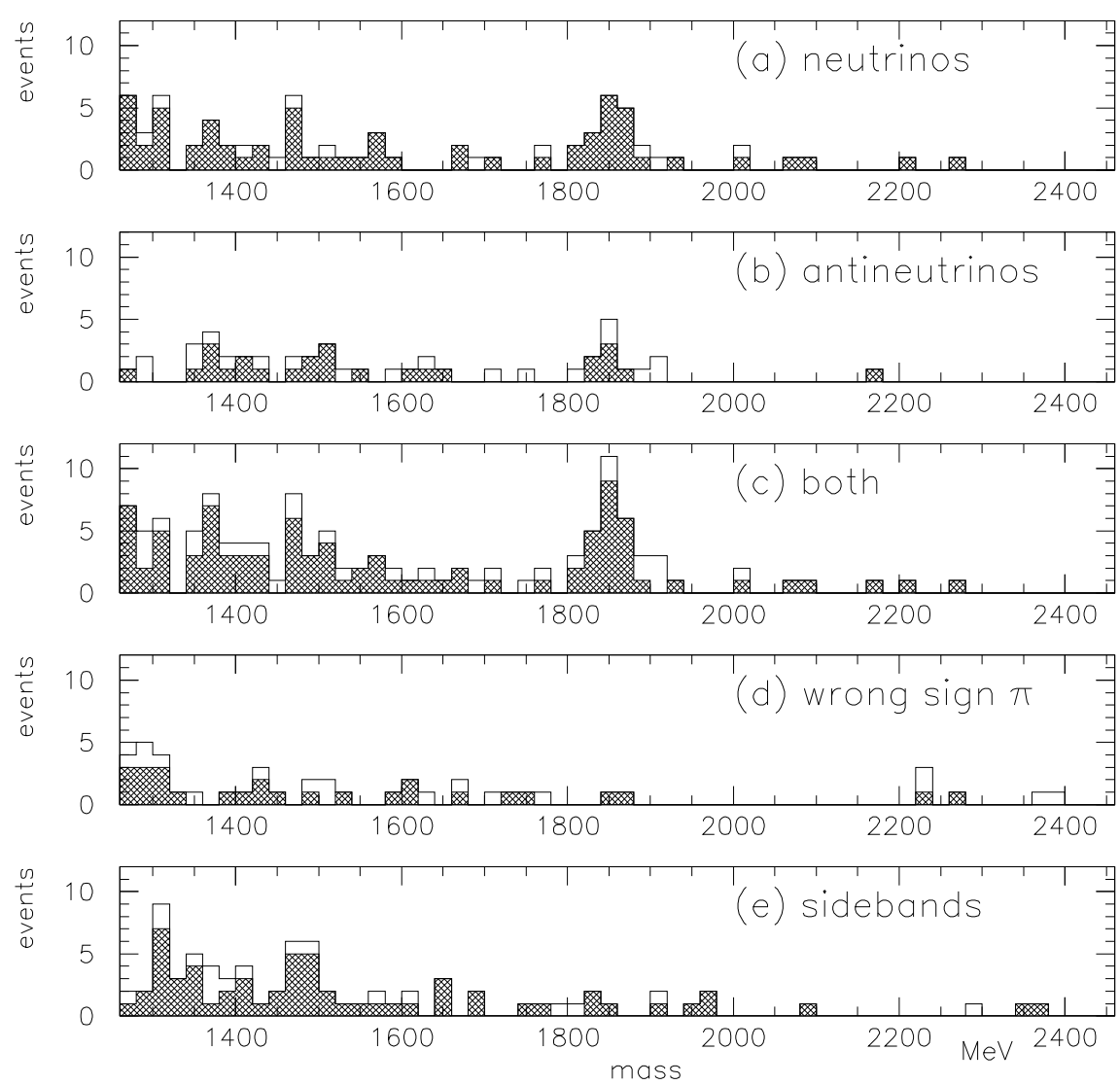

Figure 1: Mass distributions of $K^{-} \pi^{+}$and $K_{s}^{0} \pi^{+} \pi^{-}$combinations tagged by a slow pion (shaded plots are the light-fill contribution) for (a) neutrinos; (b) antineutrinos; (c) $\nu$ and $\bar{\nu}$ combined; (d) the same but with a wrong-sign slow pion; (e) the same but with the mass difference $r$ in the sidebands.

(anti)neutrino energy of the peak events for the undetected neutrals using

$$
E_{\nu}=P_{\mu}^{l}+R \cdot P_{h}^{l}
$$

Here $P_{\mu}^{l}$ is the longitudinal momentum of the muon and $P_{h}^{l}$ of the measured hadrons. $R$ is the ratio between the mean value (for the selected events) of the transverse momentum (with respect to the neutrino direction) of the muon, $P_{\mu}^{t}$, and the mean value for the detected hadrons $P_{h}^{t, i n}$ (in the neutrino-muon plane, with gammas and reinteractions of neutral particles excluded). For the peak events (within 2.5 deviations of the $D^{0}$ mass in Fig 2a) we obtain the value $R=1.22 \pm 0.16$ leading to a significantly smaller energy correction than that previously found for the inclusive charged current sample, see ref. [10]. This is compatible with the $D^{*+}$ meson carrying the bulk of the event hadronic energy, so that only the remaining bit has to be corrected.

For the mean fractional hadronic energy of the $D^{*+}$ meson in the laboratory system, we 

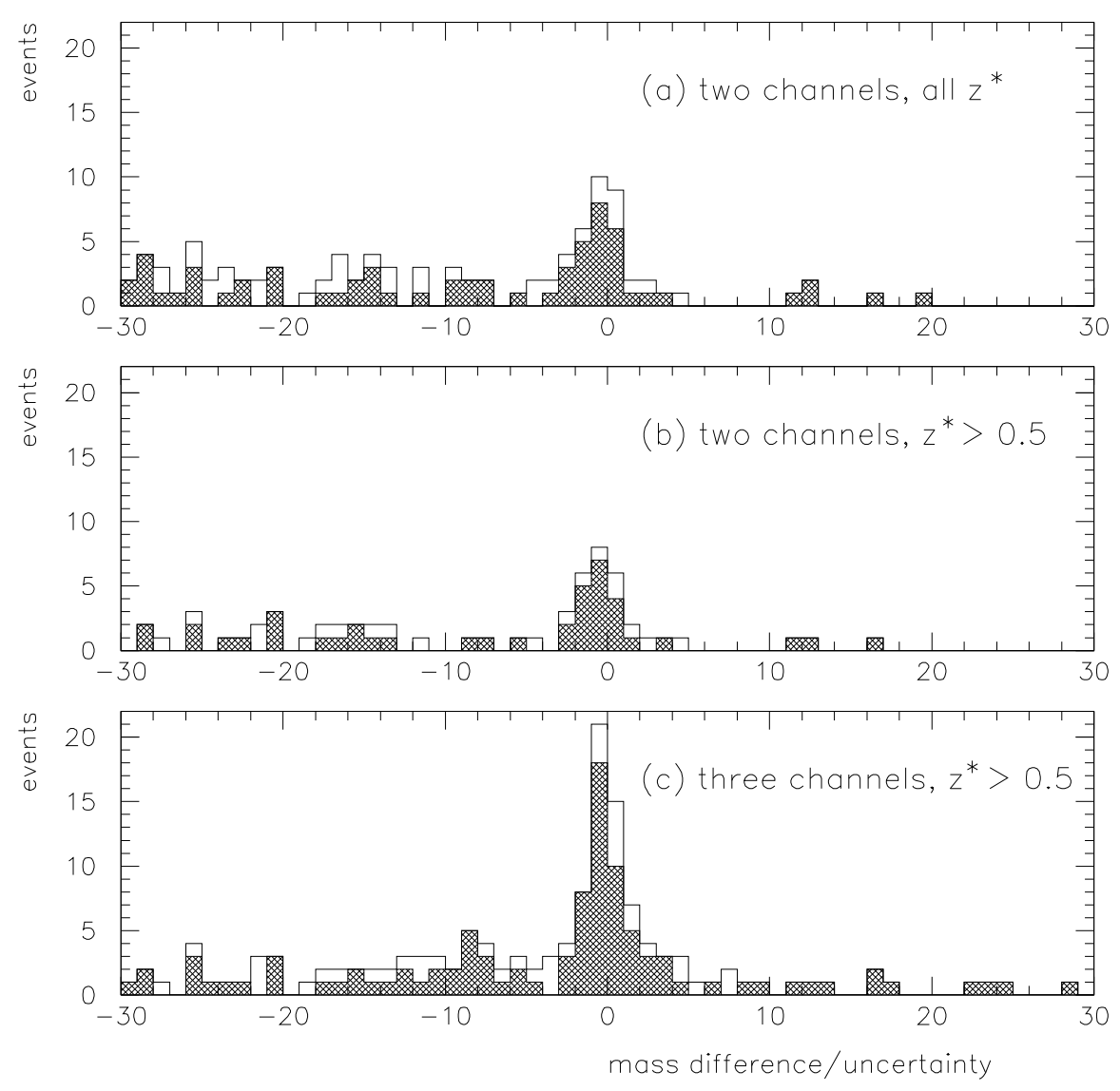

Figure 2: The 'detachment', $\left[m\left(X^{0}\right)-m\left(D^{0}\right)\right] / \sigma_{m}$, (shaded plots are the light-fill contribution) for: (a) the two channels $X^{0}=K^{-} \pi^{+}$and $K_{s}^{0} \pi^{+} \pi^{-}$(as in Fig 1c); (b) for these two channels with $z^{*}$ above $0.5 ;(\mathrm{c})$ including $K^{-} \pi^{+} \pi^{-} \pi^{+}$, with $z^{*}$ above 0.5 .

find:

$$
<z^{*}>=0.59 \pm 0.03(\text { stat }) \pm 0.08(\text { syst }) .
$$

The systematic error is estimated by increasing and decreasing the $R$ value by one standard deviation. The $\left\langle z^{*}\right\rangle$ value may be compared with $\langle z\rangle=0.61 \pm 0.04$ for the pseudoscalar $D$ mesons as measured in an emulsion experiment[11], and with $<z^{*}>$ near 0.5 for the fragmentation of a $45 \mathrm{GeV}$ charmed quark into a $D^{*+}$ meson[12],[13].

The detachment is plotted for the two channels with $X^{0}=K^{-} \pi^{+}$and $K_{s}^{0} \pi^{+} \pi^{-}$in Fig $2 \mathrm{a}$ and for the remaining events with $z^{*}$ above 0.5 in Fig $2 \mathrm{~b}$. The bulk of the signal is seen to have $z^{*}$ above 0.5 . The requirement that $z^{*}$ be above 0.5 both selects most of the $D^{*+}$ signal and also strongly suppresses the background to the $D^{0} \rightarrow K^{-} \pi^{+} \pi^{-} \pi^{+}$decays. These 4 -body decays are expected to effectively double the observed signal, but the combinatorial backgound for all $z^{*}$ is too large. Therefore the data including all three available $D^{0}$ decay channels $\left(K^{-} \pi^{+}, K_{s}^{0} \pi^{+} \pi^{-}\right.$, and $K^{-} \pi^{+} \pi^{-} \pi^{+}$) is selected with the requirement that $z^{*}$ be above 0.5 . The corresponding detachment distribution (Fig 2c) verifies that the resolution for the mass peak shown in Fig 

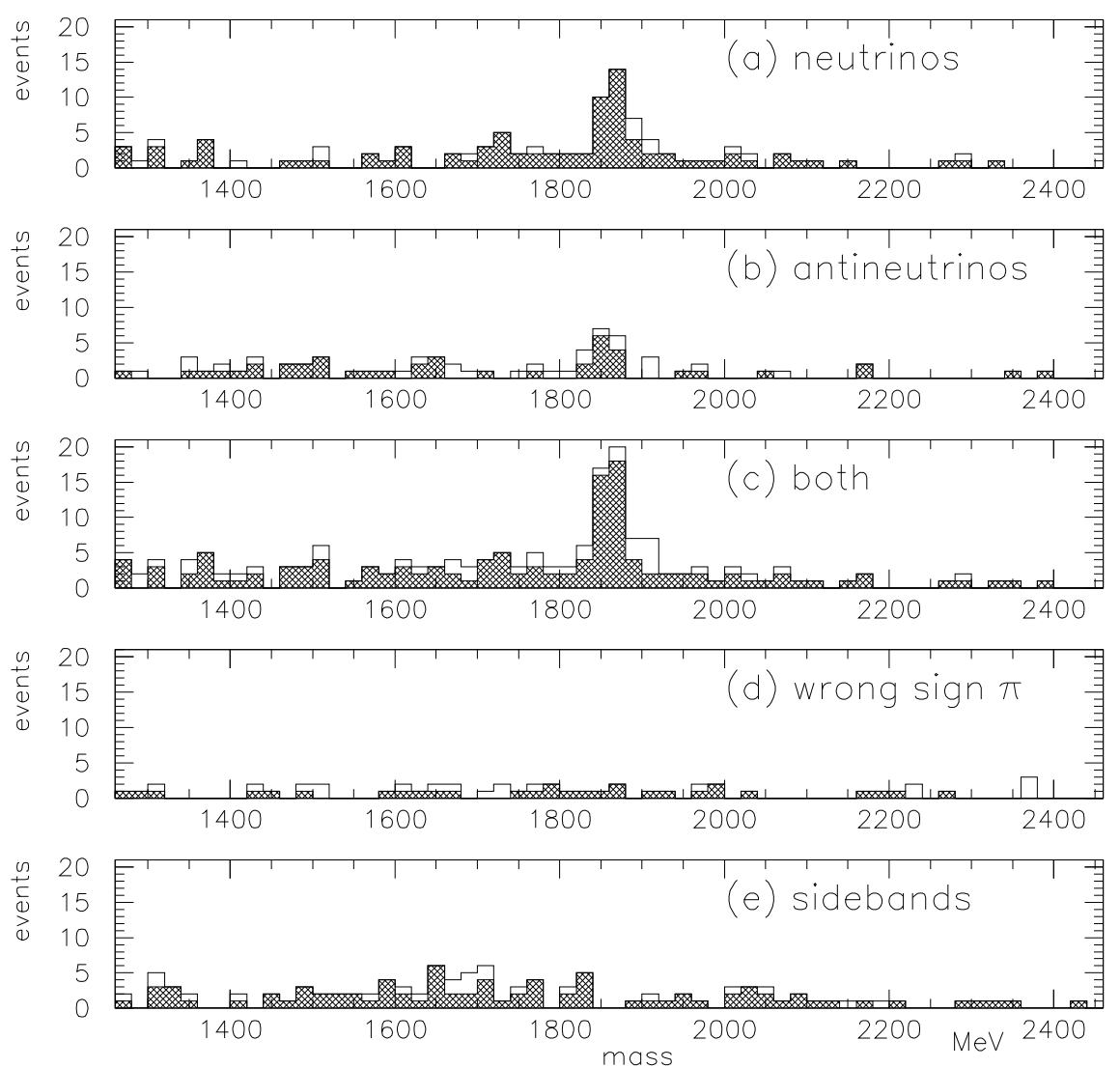

Figure 3: Mass distributions of the $X^{0}=K^{-} \pi^{+}, K_{s}^{0} \pi^{+} \pi^{-}$and $K^{-} \pi^{+} \pi^{-} \pi^{+}$combinations with $z^{*}$ above 0.5 tagged by a slow pion (shaded plots are the light-fill contribution): (a) neutrinos; (b) antineutrinos; (c) $\nu$ and $\bar{\nu}$ combined; (d) the same but with a wrong-sign slow pion; (e) the same but with the mass difference $r$ in the sidebands.

$3 \mathrm{c}$ is satisfactory. The light-fill contribution to the peak has a smaller width (shaded parts of Figures 2 and 3). The corresponding wrong-sign and wrong-difference backgrounds (Figures $3 \mathrm{~d}$ and $3 \mathrm{e}$ ) are featureless. (A higher level of the latter background is due to a steep increase of the phase space toward higher values of the mass difference.)

For the neutrino and antineutrino components of the peak in figure 3 (with $z^{*}$ above 0.5 ), we obtain $\left\langle x_{B j}\right\rangle=0.20 \pm 0.03$ and $0.11 \pm 0.02$, respectively, suggesting production from sea quarks by antineutrinos. The mean neutrino and antineutrino energies in the peak are $70.0 \pm 8.6$ and $60.6 \pm 8.2 \mathrm{GeV}$, respectively, exceeding the means for all charged current events as expected for charmed particle production.

For the determination of the $D^{*+}$ production cross-section, the overall $z^{*}$ range is split into the events with $z^{*}$ below 0.5 and those with $z^{*}$ above 0.5. For the former, only the $D^{0} \rightarrow K^{-} \pi^{+}, K_{s}^{0} \pi^{+} \pi^{-}$decays are considered. For $z^{*}$ above 0.5 , all three $D^{0}$ decay modes are used, see Fig 3 . The corresponding detachment plots are fitted to a sum of a linear background and a Gaussian with the position fixed at zero and width fixed to unity. (The latter values 

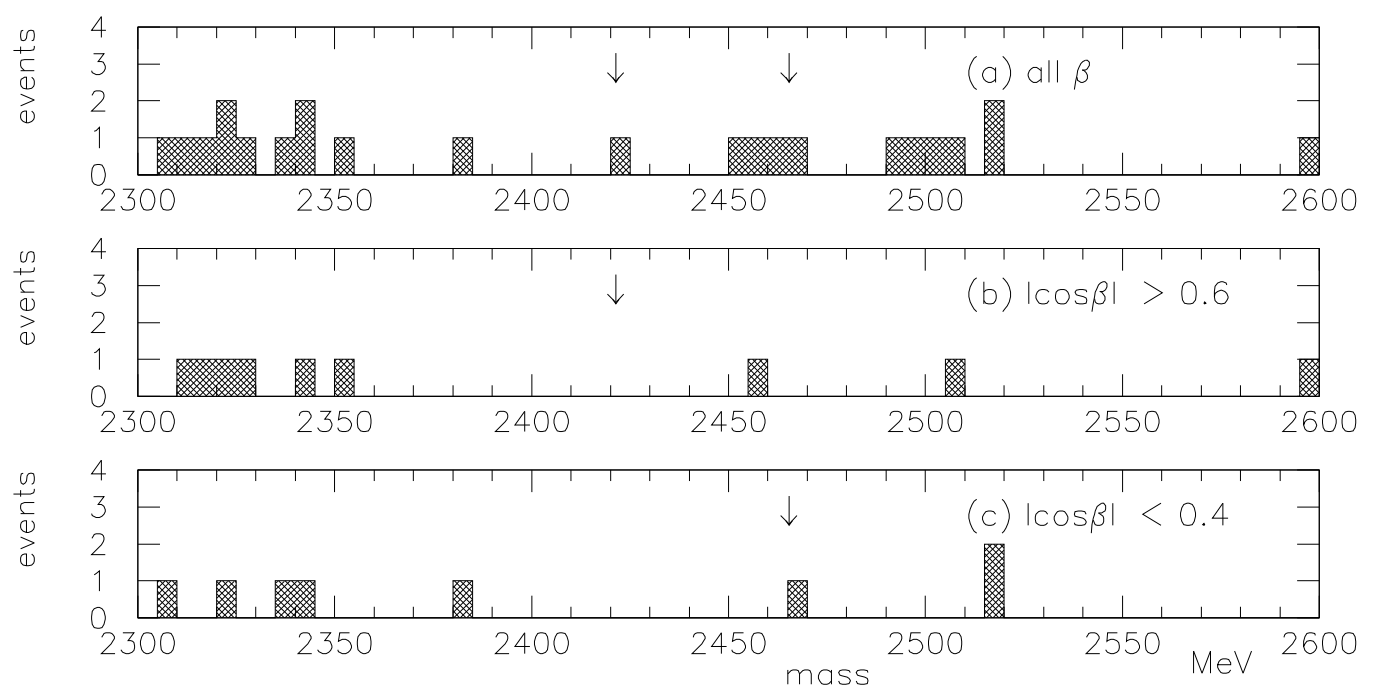

Figure 4: Distribution of the mass, $m\left(X^{0} \pi^{+} \pi^{-}\right)-m\left(X^{0} \pi^{+}\right)+m\left(D^{*+}\right)$, of the $D^{*+} \pi^{-}$candidates with $z^{* *}$ above 0.5 , (with $m\left(X^{0}\right)$ within 2.5 deviations of $m\left(D^{0}\right)$ and $X^{0} \pi^{+}$passing the $D^{*+}$ selection): (a) no helicity angle selection (see text); (b) for the $D^{* * 0}(2420)$ with $|\cos \beta|>0.6$; (c) for the $D^{* * 0}(2460)$ with $|\cos \beta|<0.4$. The arrows indicate the $D^{* * 0}$ masses from reference 5 .

are compatible with the results of a fit treating them as free parameters). The dominant contribution to the cross-section comes from the $z^{*}$ above 0.5 region. The fit to Fig $2 \mathrm{c}$ gives $45.6 \pm 7.4$ events in the peak between -2.5 and 2.5 and a background of $8.4 \pm 1.1$ events. A similar fit to Fig $3 \mathrm{c}$ gives the mass of the $D^{0}$ to be $1862 \pm 3 \mathrm{MeV}$.

The $D^{*+}$ production rates per charged current interaction with an isoscalar target are estimated as $(1.22 \pm 0.25) \%$ and $(1.01 \pm 0.31) \%$ for the neutrinos and antineutrinos, respectively. Here the measured $D^{0}$ and $D^{*+}[14]$ branching fractions are used. The rates are corrected for the non-isoscalarity of the hydrogen target whereby the neutrino and antineutrino production rates are effectively decreased and increased, respectively, by about $9 \%$. The signal in the neon contribution is corrected for the loss due to the fractional momentum error cut. Our neutrino rate agrees with the previous measurement of $\left(0.99_{-0.44}^{+0.52}\right) \%[3]$ using the WA21 data included in this analysis, but not the $D^{0} \rightarrow K_{s}^{0} \pi^{+} \pi^{-}$decays. The antineutrino production rate is lower than $(4.2 \pm 1.3) \%$ as reported in an early measurement[2] by 2.3 standard deviations.

\section{4. $D^{* * 0}$ production.}

The search for $D^{* * 0} \rightarrow D^{*+} \pi^{-}$decays uses $D^{*+}$ with all three available $D^{0}$ decay channels. We form the $\left(X^{0} \pi^{+}\right) \pi^{-}$combinations scanning the remaining $\pi^{-}$mesons, with the $X^{0} \pi^{+}$combination passing the mass difference selection and $m\left(X^{0}\right)$ within 2.5 standard deviations of the $D^{0}$ mass. The fractional hadronic energy of the $\left(X^{0} \pi^{+}\right) \pi^{-}, z^{* *}$, is required to be above 0.5 , which is a similar selection to $z^{*}$ above 0.5 for the reference $D^{*+}$ sample. Fig 4a shows the 
'precision' mass,

$$
m^{\prime}\left(X^{0} \pi^{+} \pi^{-}\right)=m\left(X^{0} \pi^{+} \pi^{-}\right)-m\left(X^{0} \pi^{+}\right)+m\left(D^{*+}\right)
$$

This is used because the precision achieved for $m^{\prime}$ is as good as some $\pm 7 \mathrm{MeV}$ due to the cancellation of most measurement errors, and is less than the natural widths of the $D^{* * 0}$ states.)

Some candidates in fig $4 \mathrm{a}$ are observed near both the $1^{+}(2420)$ and $2^{+}(2460)$ masses. To reduce the background, we use the 'helicity angle', $\beta$, between the $D^{* *}$ daughter $\pi^{-}$and the $D^{*}$ daughter $\pi^{+}$, as measured in the $D^{*}$ rest frame. With both $1^{+}$and $2^{+}$decaying into $D^{*} \pi$ in the $\mathrm{d}$-wave, the distribution in $\cos \beta$ behaves like $\left(1+3 \cos ^{2} \beta\right)$ for the $1^{+}(2420)$ decays, and $\sin ^{2} \beta$ for the $2^{+}(2460)$ decays[5]. The selection $|\cos \beta|>0.6$ used in Fig $4 \mathrm{~b}$ emphasizes the $1^{+}(2420)$ production (acceptance 0.59 ), while the selection $|\cos \beta|<0.4$ used in Fig 4c is aimed at $2^{+}(2460)$ production (acceptance 0.57$)$. Under these selections, we are left with no $1^{+}(2420)$ candidate and a single $2^{+}(2460)$ candidate. (The latter is an antineutrino event in deuterium with the possible decay chain $D^{* * 0} \rightarrow D^{*-} \pi^{+}, D^{*-} \rightarrow D^{0} \pi^{-}$and $D^{0} \rightarrow K^{-} \pi^{+}$with $m\left(X^{0}\right)=1875 \pm 20 \mathrm{MeV}$, mass difference $r=6.9 \pm 0.8 \mathrm{MeV}$, and precision mass $m^{\prime}=2466 \pm 7$ $\mathrm{MeV}$.

The fit to fig $2 \mathrm{c}$ yielded a signal of $45.6 \pm 7.4$ observed $D^{*+}$ decays in the reference sample, from which we obtain the two following $90 \%$ confidence level upper limits. The number of $D^{* * 0}(2420)$ with $z^{* *}$ above 0.5 is less than $10 \%$ of the number of $D^{*+}$ with $z^{*}$ above 0.5 . With the same $z$ selections, the number of $D^{* * 0}(2460)$ is less than $18 \%$ of the number of $D^{*+}$. For the likely assumption that the $z$ distribution of the $D^{* *}$ is not softer than that of directly produced $D^{*}$, these upper limits apply to the fractions of all produced $D^{*+}$ mesons which could be daughters of $D^{* * 0}(2420)$ and $D^{* * 0}(2460)$, respectively.

Further assuming that the ratios of $D^{* * 0}$ to $D^{*+}$ production are equal for neutrinos and antineutrinos and using their respective charged $D^{*}$ production cross-sections as estimated above, the $90 \%$ confidence level upper limits for the $D^{* * 0}(2420)$ production rate per charged current interaction times the $D^{* * 0}(2420) \rightarrow D^{*+} \pi^{-}$branching fraction are $0.15 \%$ for neutrinos and $0.13 \%$ for antineutrinos. The corresponding upper limits for $D^{* * 0}(2460)$ are $0.27 \%$ and $0.24 \%$, respectively.

Alternative $D^{* * 0}(2420)$ decay channels include $D^{* 0} \pi^{0}$ and, possibly, various $D \pi \pi$ and $D^{*} \pi \pi$ final states (note that the $1^{+} \rightarrow D \pi, D \eta$ decays are forbidden by parity). If $D \pi \pi$ and $D^{*} \pi \pi$ decays can be neglected, the above upper limits translate into those of less than $0.23 \%$ (neutrinos) and $0.20 \%$ (antineutrinos) for the $D^{* * 0}(2420)$ production rate per charged current interaction.

Surprisingly, our study[4] based on the same experimental data indicates that the strange counterpart of $D^{* * 0}(2420)$, the heavier $D_{s}^{* *+}(2536)$ meson, is produced more abundantly by neutrinos than are the $D^{* * 0}$ : with a lower neutrino energy cut of $10 \mathrm{GeV}$ for comparison with the present analysis instead of $20 \mathrm{GeV}$ as in reference [4], as well as updated $D^{0}$ branching fractions[14], the $D_{s}^{* *}(2536)$ rate per charged current interaction is estimated as $(0.85 \pm 0.38) \%$ for neutrinos (with 8 events seen), and less than $0.40 \%$ (at $90 \%$ confidence level) for antineutrinos where only one event was found.

\section{Conclusions.}

From the combination of data from three high-statistics neutrino experiments using BEBC and the CERN wide band neutrino beam we conclude:

- The rates of charged $D^{*}$ meson production per charged current neutrino and antineutrino interaction on an isoscalar target are $(1.22 \pm 0.25) \%$ and $(1.01 \pm 0.31) \%$, respectively.

- The mean fractional hadronic laboratory energy of the charged $D^{*}$ is $0.59 \pm 0.03 \pm 0.08$.

- No candidate is found for $D^{* * 0}(2420)$ and only one possible candidate for $D^{* * 0}(2460)$. Upper 
limits of $10 \%$ and $18 \%$ (at the $90 \%$ confidence level) are found for the fractions of all produced charged $D^{*}$ mesons which come from $D^{*} \pi$ decays of the $D^{* * 0}(2420)$ and $D^{* * 0}(2460)$ mesons, respectively. We conclude that no more than $18 \%$ of all $D^{*+}$ mesons produced in (anti)neutrino interactions come from the $D^{* * 0}$ decays at these neutrino energies, while at LEP this fraction was measured as $(18 \pm 5 \pm 2) \%[12]$.

Acknowledgements. We would like to thank the WA21, WA25 and WA59 collaborations for allowing us to include their data.

\section{References}

[1] J. Blietschau et al., Phys. Lett. 86B, 108 (1979).

[2] A.E. Asratyan et al. (E180), Phys. Lett. 132B, 246 (1983)

[3] G.T. Jones et al. (WA21), Z. Phys. C36, 593 (1987)

[4] A.E. Asratyan et al., Z. Phys. C61, 563 (1994)

[5] G. Crawford et al. (CLEO), Phys. Lett. B331, 236 (1994)

[6] G.T. Jones et al. (WA21), Z. Phys. C51, 11 (1991)

[7] D. Allasia et al. (WA25), Z. Phys. C37, 527 (1988)

[8] K. Varvell et al. (WA59), Z. Phys. C36, 1 (1987)

[9] J. Adler et al. (Mark III), Phys. Rev. Lett. 64, 2615 (1990)

[10] M. Aderholz et al., Phys. Lett. 173B, 211 (1986)

[11] N. Ushida et al. (E531), Phys. Lett. 121B, 292 (1983)

[12] D. Buskulic et al. (ALEPH), Z. Phys. C62, 1 (1994)

[13] P. Abreu el al. (DELPHI), Z. Phys. C59, 533 (1993);

R. Akers et al, (OPAL), CERN-PPE 94-217, submitted to Z. Phys. C.

[14] Particle Data Group, Phys. Rev. D50, no. 3 (1994) 